\title{
A Comparative CFD Study of Side-view Mirror and Side-view Camera Usages on a City Bus
}

\author{
Duygu Ipci ${ }^{1}$ \\ 0000-0002-8862-7662 \\ ${ }^{1}$ Automotive Engineering Department, Faculty of Technology, Gazi University, Ankara, 06500, Turkey
}

\begin{abstract}
In this study, the effects of classical side mirrors and side-view cameras on the aerodynamic drag coefficient of a city bus model were investigated with Computational Fluid Dynamics (CFD). The surface profile of 1/5 scale bus was modeled with CATIA v5 program. Aerodynamic analyses were performed with ANSYS Fluent ${ }^{\circledR}$ v18. Reynolds Average Navier-Stokes (RANS)-based Reynolds Stress Model (RSM) was used as the turbulence model. The ground effect was taken into consideration in all analyses. The solution domain for $5 \%$ blockage ratio was created. Inflation layers are used on the vehicle surface regarding velocity changes due to viscous effects. The mesh number independence of analyses was examined for the bus model without side mirrors and provided for 1.3 M mesh element. The Reynolds number independence of the analysis was investigated by using the optimum number of mesh. Free flow velocity of 90 $\mathrm{m} / \mathrm{s}$ and above were found to be adequate for Reynolds number independence. The drag coefficient was calculated for the bus with side mirrors by using optimum mesh and wind speed as well. The drag coefficients for the bus with side mirrors and side view cameras were determined 0.539 and 0.521 respectively.
\end{abstract}

Keywords: Drag coefficient, Reynolds Stress Model, side view mirror, side view camera, passive flow control

\author{
* Corresponding author \\ Duygu Ipci \\ duyguipci@gazi.edu.tr
}

Adress: Automotive Engineering Department, Faculty of Technology, Gazi University, Ankara, Turkey

Tel:+903122028639

Fax: +903122028639

Research Article

$\begin{array}{ll}\text { Manuscript } & \\ \text { Received } & 24.04 .2020 \\ \text { Revised } & 12.06 .2020 \\ \text { Accepted } & 01.07 .2020\end{array}$

Doi: 10.30939/ijastech..726376

\section{Introduction}

Aerodynamics is a branch of physics that studies the interaction of solid bodies moving with air. When a fluid moves on a solid body, it applies pressure forces perpendicular to the surface and shear forces parallel to the surface along the outer surface of the body. In aerodynamics, instead of the details of the distributions of pressure and shear forces along the entire surface of the body, the combination of these forces are concerned. The component of the resultant pressure and shear forces in the flow direction is called the drag force, and the component that acts perpendicularly in the direction of the flow is called the lift force. The properties of the fluid flow around the parts, such as the mirror, the wheel, and the lids on the bus, play an important role in increasing aerodynamic drag. These parts should be designed regarding the fluid flow around the bus. Aerodynamic analyses are carried out to control the change of drag by taking precautions against drag force and power losses caused by the acceleration of vehicles. Increasing accurate analysis results over the years have prompted vehicle manu- facturers to produce lower drag vehicle bodies.

To improve the aerodynamic performance of road vehicles, various drag reduction methods have been used. These methods can be classified as passive or active flow control methods. Passive control methods generally include the geometrical modifications such as splitter plates, splitter wedges, base bleed, boat-tailing and various types of serrated trailing edges. Active flow control methods, on the other hand, involve energy or momentum addition to the flow in a regulated manner. The most used and oldest drag reduction methods are passive flow control methods and do not consume external energy. Wang et.al [1] presented the novel drag reduction method for automotive mirrors using passive jet flow control. This method found to be effective both wind tunnel testing with PIV and CFD large eddy simulation (LES) calculation. Gan et.al. [2] conducted a study on reducing the truck rearview mirror drag using passive flow control jet boat tail (JBT) technique. They reported that the wind tunnel testing measured a drag reduction of $10.1 \%$ due to the JBT configuration and the 3D CFD predicts a drag reduction of $11.0 \%$. Roy et al. [3] were geometrically modi- 
fied the front and rear parts of a bus and tested them with CFD simulations. They reported that the tapering of the back and front of the bus and the rounding of the sharp edges reduce aerodynamic drag and contributes to fuel economy.

Ipci et al.[4] investigated the flow pattern around a simplified vehicle model which is called as Ahmed body by using CFD. In the CFD simulations, $\mathrm{k}-\varepsilon$ and RNG k- $\varepsilon$ turbulence models were used to examine the vortexes on the back of the model. The numerical results were compared to a publication results in which an experimental study was conducted and the RNG k- $\varepsilon$ turbulence model found to be more consistency with experimental results. Gebel et al. [5] tested a 1/64 scale electric vehicle prototype at different flow rates and observed the change in the drag coefficient $\left(C_{D}\right)$. As a result of the experiments, they observed that the change was less than $1 \%$, that is, independent of the Reynolds number.

In recent years, various studies have been carried out on the use of cameras instead of the side mirrors. The side view mirrors ratio is $2.5-5$ percent of the overall aerodynamic drag of the vehicle [6]. Therefore, it is recommended to use cameras that are smaller in size than the side mirrors. Hirose et.al.[7] studied on the interference drag that is generated by the mixing of airflow streamlines between door mirrors and vehicle body. Authors derived an equation that could be predicted interference drag by utilizing between the functions of velocities around the vehicle body and door mirror drag. The values calculated by the newly derived equation were compared with the measured data obtained from wind tunnel tests. The results were found to be a good agreement with experiments. Buscariolo and Rosilho [8] conducted a two-stage CFD study to examine the effect of mirror and camera uses on drag coefficients for side view in cars. In the first stage, they made a comparison between the drag coefficients of a vehicle with and without a side view mirror. In the second stage, two video camera housing proposals were evaluated for replacing of a side view mirror and their drag coefficients were compared with baseline car. It was reported that camera housing design that bonded to vehicle body was improved drag coefficient reduction of $1 \%$, similar to the case without mirrors.

The aim of this paper is to investigate drag coefficient of a city bus by putting the place of the side-view mirrors for cameras placed in housing.

\section{Material and Method}

In this study, a 1/5 scale city bus was modeled and aerodynamic analysis was performed with ANSYS Fluent ${ }^{\circledR}$. The $1 / 5$ scale model of the city bus was created with the CATIA and the front and side views of the bus dimensions are given in Figure 1. Drag coefficient of the bus without side mirror was calculated to examine the effects of the side mirrors and cameras on the aerodynamic drag.
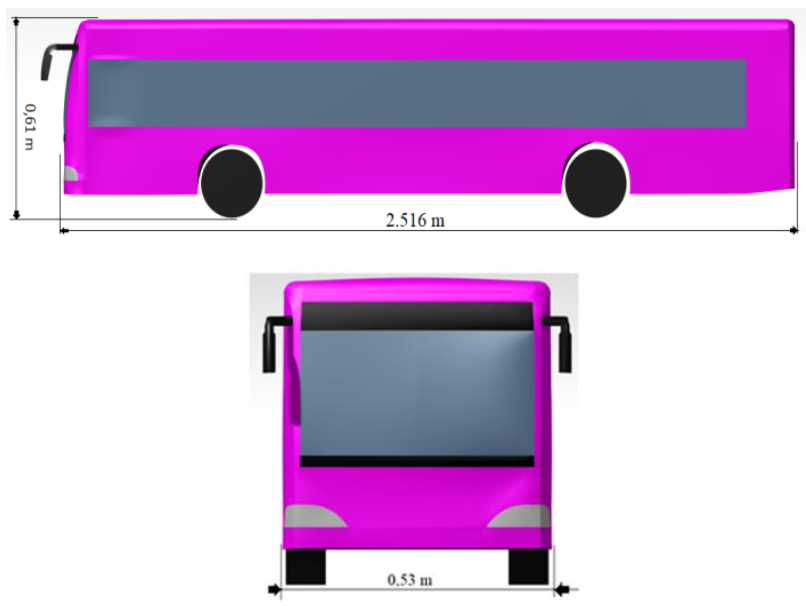

Fig. 1. Front and side views of the bus

During the CFD analysis of the model bus, a control volume was created by using the appropriate blockage ratio for a continuous set of streamlines on solution domain boundaries. The blockage ratio is defined as the frontal area of the object/cross-section of the wind tunnel. The minimum blockage ratio in wind-tunnel testing is required to be $4 \%$ [9]. In this study, the blocking ratio was used as 5\% and the dimensions of the cross-section of the control volume perpendicular to the flow are determined as $2.4 \mathrm{~m} \mathrm{x} 2.8 \mathrm{~m}$. The distance of the front of the vehicle from the control volume is 0.75 times the length of the vehicle, and the distance of the rear is 1.5 times the length of the vehicle.

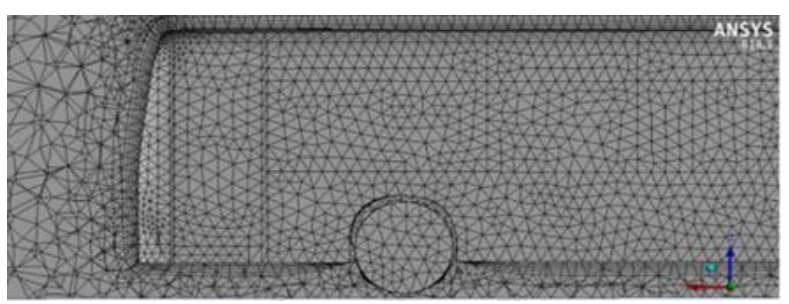

Fig. 2. The mesh structure of solution domain

As seen in Figure 2, tetrahedral mesh structure is used on the solution domain. Since the speed changes due to viscous effects on the surface of the bus will be high, the inflation layers are created on whole surface of the body. The mesh structure is divided into small element sizes by utilizing the body sizing control. In the investigation of mesh independence, inflation layers has been kept constant and CFD analyses have been conducted with changing the mesh element size of solution domain and surface of the vehicle body. 
Table 1. Side mirror and proposed camera housing

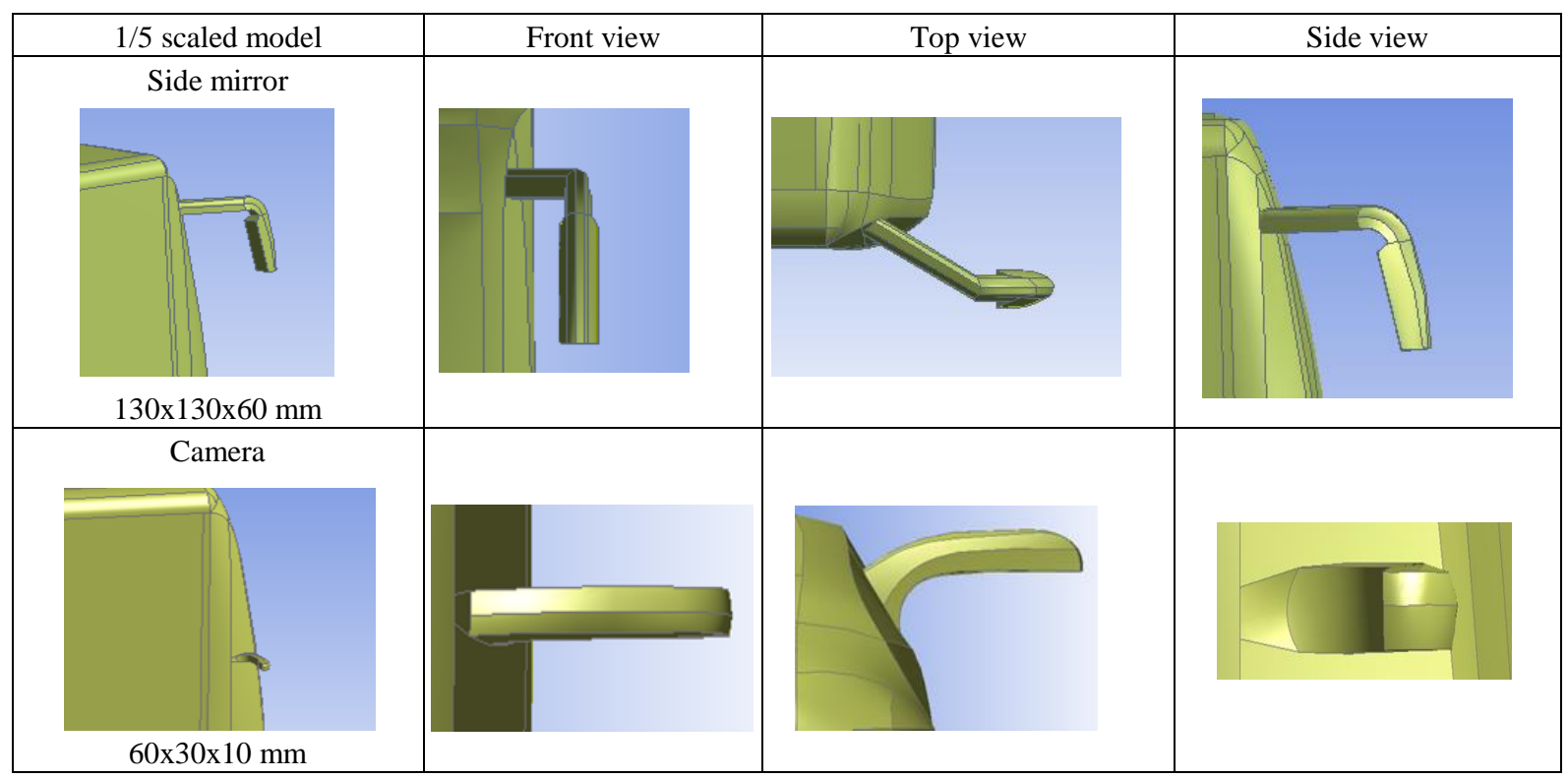

In Table 1. side mirror and proposed camera housing are indicated. Dimensions of the side mirror and proposed camera housing of 1/5 scaled bus are 130x130x60 mm and $60 \times 30 \times 10 \mathrm{~mm}$ respectively. Dimensions of the camera, used as reference for housing modeling, are approximately $35 \mathrm{~mm}$ length and diameter of $25 \mathrm{~mm}$. A camera housing are designed and positioned to fit the bus regarding the camera dimensions.

\subsection{Turbulence Model}

The turbulence model has been chosen based on the results of a published study [10]. In the published study, the drag coefficient for Ford $\mathrm{K}$ passenger car obtained from wind tunnel test were compared with the results calculated by realizable $k-\varepsilon$ and Reynolds Stress (RSM) turbulence models. It is stated that the drag coefficient obtained from the RSM turbulence model approaches shows excellent agreement with the experimental results. In this study RSM turbulence model was chosen for use in CFD analysis.

The ANSYS Fluent ${ }^{\circledR}$ generally performs turbulent flow analysis with RANS based turbulence models. In RANS turbulence models, continuity and momentum equations are rearranged by Reynolds decomposition and time averages, and this method shows statistical properties. In derivation of RANS equations, firstly, terms of turbulent flow quantities such as $\mathrm{u}, \mathrm{v}, \mathrm{w}$ and $\mathrm{p}$ were decomposed average and fluctuating components. For example, instantaneous $\mathrm{u}$ velocity is decomposed in the RANS model as $u=\bar{u}+u^{\prime}$. Terms of $\bar{u}$ and $\bar{u}$ shows the mean velocity and fluctuations of instantaneous $\mathrm{u}$ velocity within time $\Delta t$. Then the time average of turbulent flow quantities is taken. As a result of these operations, continuity equation and general momentum equations for incompressible flows may be stated as respectively;

$$
\begin{aligned}
& \bar{u}_{i, j}=0 \\
& \frac{\partial \bar{u}_{i}}{\partial t}+\bar{u}_{j} \frac{\partial \bar{u}_{i}}{\partial x_{j}}=-\frac{1}{\rho} \frac{\partial \bar{p}}{\partial x_{i}}+\frac{1}{\rho} \frac{\partial}{\partial x_{j}}\left[\begin{array}{l}
\left.\mu\left(\frac{\partial \bar{u}_{i}}{\partial x_{j}}+\frac{\partial \bar{u}_{j}}{\partial x_{i}}\right)\right] \\
-\rho \overline{u_{i}^{\prime} u_{j}^{\prime}}
\end{array}\right]
\end{aligned}
$$

The term $\rho \overline{u_{i}^{\prime} u_{j}^{\prime}}$ given in the Equation (2) shows the stresses caused by turbulence and is called as Reynolds stress tensor. RANS based turbulence models are solved through the Eddy viscosity concept or using transport equation models. Disregarding the isotropic eddy-viscosity hypothesis, the RSM closes the Reynolds-averaged Navier Stokes equations by solving transport equations for the Reynolds stresses, together with an equation for the dissipation rate. RSM method used in this study is the second order differential equation model with 7 equations that is, the transport equations are solved in 3D [11]. In second order models, Reynolds stresses and turbulence flows (second order moments) are calculated by using conservation equations directly instead of Boussinesq approach.

Figure 3 shows the sub-options and equation constants selected in the RSM turbulence method. In the analysis, linear pressure-strain, standard wall functions and standard equation constants given in Fluent ${ }^{\circledR}$ program were used. 
2. Viscous Model

\begin{tabular}{|c|c|}
\hline \multirow{2}{*}{$\begin{array}{l}\text { Model } \\
\text { Inviscid }\end{array}$} & Model Constants \\
\hline & Cmu \\
\hline Laminar & 0.09 \\
\hline \multirow{2}{*}{$\begin{array}{l}\text { Spalart-Allmaras ( } 1 \text { eqn) } \\
\text { k-epsilon ( } 2 \text { eqn) }\end{array}$} & C1-Epsilon \\
\hline & 1.44 \\
\hline$\left\{\begin{array}{l}\text { k-omega ( } 2 \text { eqn) } \\
\text { Transition k-kl-omega ( } 3 \text { eqn) }\end{array}\right.$ & C2-Epsilon \\
\hline \multirow{2}{*}{$\begin{array}{l}\text { Transition SST (4 eqn) } \\
\text { Reynolds Stress (7 eqn) }\end{array}$} & 1.92 \\
\hline & C1-PS \\
\hline \multirow{3}{*}{$\begin{array}{l}\text { Scale-Adaptive Simulation (SAS) } \\
\text { Detached Eddy Simulation (DES) } \\
\text { Large Eddy Simulation (LES) }\end{array}$} & 1.8 \\
\hline & C2-PS \\
\hline & 0.6 \\
\hline \multirow{4}{*}{$\begin{array}{l}\text { Reynolds-Stress Model } \\
\text { Linear Pressure-Strain } \\
\text { Quadratic Pressure-Strain } \\
\text { Stress-Omega } \\
\text { stress-BSL }\end{array}$} & C1'-PS \\
\hline & 0.5 \\
\hline & C2'-PS \\
\hline & 0.3 \\
\hline \multirow{3}{*}{$\begin{array}{l}\text { Reynolds-Stress Options } \\
\begin{array}{l}\square \text { Wall BC from k Equation } \\
\square \text { Wall Reflection Effects }\end{array}\end{array}$} & TKE Prandtl Number \\
\hline & 1 \\
\hline & TDR Prandtl Number \\
\hline Near-Wall Treatment & 1.3 \\
\hline $\begin{array}{l}\text { Standard Wall Functions } \\
\text { Scalable Wall Functions } \\
\text { Non-Equilibrium Wall Functions } \\
\text { Enhanced Wall Treatment }\end{array}$ & \\
\hline
\end{tabular}

Fig. 3. Sub-options and constants of RSM turbulence model

\subsection{Boundary Conditions}

Figure 4 indicates boundary conditions of the solution domain. The wall boundary condition is defined for the bottom surface of solution domain because of that the vehicle is practically on the road. The symmetry condition is used to disregard shear stresses on the right, left and top sides of the solution domain. The wall boundary condition is defined on the entire surface of the bus.

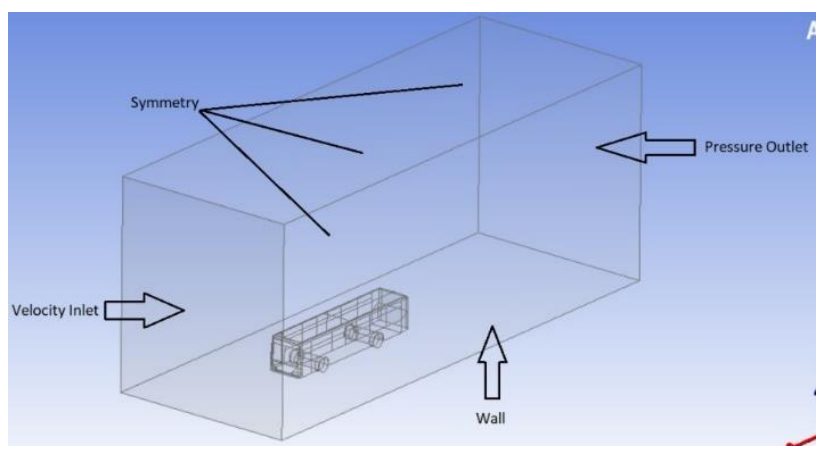

Fig. 4. Solution domain and boundary conditions

\subsection{Mesh Independency and Mesh Quality}

Sufficient number of mesh is required for the accuracy and stability of the analysis. While insufficient number of mesh usage causes errors in analysis, the use of more than optimum level causes unnecessary process time. For this reason, mesh independence of the solution needs to be investigated. In this study, using the tetrahedral mesh structure, the mesh independence of the analysis was investigated with $10 \mathrm{~m} / \mathrm{s}$ fluid velocity for bus model without side view mirrors. Table 2 gives the drag coefficients calculated for the nodes and element quantities used in all analyses. It is seen that the mesh structure with 1.3 million elements is sufficient. In the analyses, where the Fluent solver is used, the mesh quality values of skewness, aspect ratio, and growth rate might be maximum as $0.9,40$, and 1.2 for the tetrahedral mesh. In this study, these mesh quality limitations are regarded.

Table 2. Calculated $\mathrm{C}_{\mathrm{D}}$ values depending on number of nodes and elements

\begin{tabular}{|r|c|c|}
\hline Node & Element & Drag coefficient, $C_{D}$ \\
\hline 130397 & 575098 & 0,54 \\
\hline 194418 & 839638 & 0,52 \\
\hline 261519 & 1090081 & 0,51 \\
\hline $\mathbf{2 9 9 9 0 5}$ & $\mathbf{1 3 0 0 4 0 0}$ & $\mathbf{0 , 5 0 8}$ \\
\hline 326280 & 1441804 & 0,508 \\
\hline
\end{tabular}

\subsection{Reynolds Independency}

After the mesh independence obtained as a result of the calculations, Reynolds independence should be investigated. While performing the process, the optimum mesh element quantity is used and inlet velocity is increased until the drag coefficient is fixed. The $C_{D}$ coefficients were determined by increasing the inlet velocity with $10 \mathrm{~m} / \mathrm{s}$ intervals starting from $10 \mathrm{~m} / \mathrm{s}$. After $90 \mathrm{~m} / \mathrm{s}$ inlet velocity there was no significant increase in $C_{D}$ coefficient and this speed was found suitable for all analysis. Table 3 indicates the $C_{D}$ coefficients of bus without side-view mirror according to the velocity.

Table 3. Calculated $C_{D}$ values depending on velocity

\begin{tabular}{|c|c|}
\hline Inlet velocity & Drag coefficient, $\mathrm{C}_{\mathrm{D}}$ \\
\hline 10 & 0,508 \\
\hline 30 & 0,506 \\
\hline 50 & 0,504 \\
\hline 70 & 0,5 \\
\hline $\mathbf{9 0}$ & $\mathbf{0 , 5 0 3}$ \\
\hline 100 & 0,503 \\
\hline
\end{tabular}

\section{Results and Discussions}

In Figure 5, the velocity contours in the direction of airflow was shown on a sectional area that longitudinally divides control volume into equal. In Figure 5, the analysis was carried out for the $10 \mathrm{~m} / \mathrm{s}$ inlet velocity and it can be seen that the fluid velocity at the boundaries of the control volume except the bottom is the same as the free stream velocity. As a result, it is understood that the created control volume dimensions are adequate to perform the analysis correctly. 


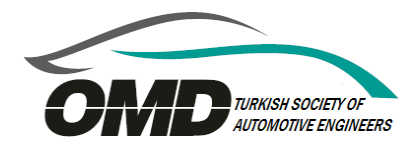

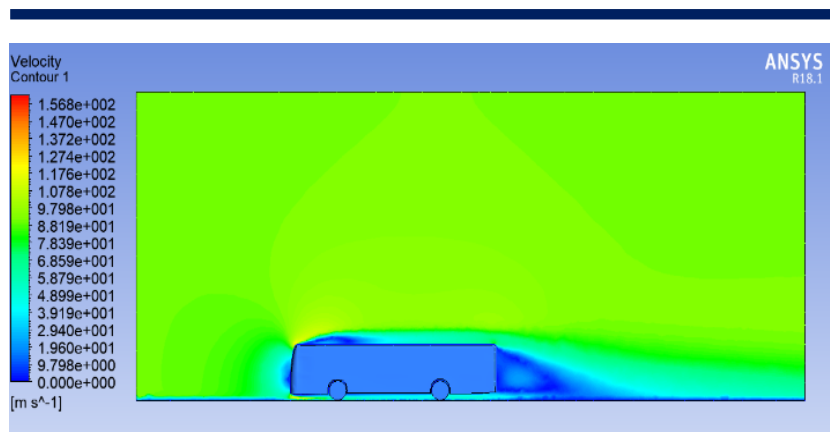

Fig. 5. Velocity contours on flow direction

Aerodynamic analysis was carried out for bus models without mirror, with mirror and camera by using the $1.3 \mathrm{M}$ element number and $90 \mathrm{~m} / \mathrm{s}$ inlet velocity. Drag coefficients due to pressure loss and viscous effects are given in Table 4. Drag coefficients were calculated as $0.503,0.539$ and 0.521 for bus models without mirror, with mirror and camera, respectively. Because of the large cross-sectional area of the side mirrors, high pressure areas were formed on the mirrors housing, it was observed that the wind resistance coefficients increased due to both pressure difference and viscous effects. Drag coefficient of the bus model with camera decreased by $3.4 \%$ compared to the bus model with side view mirror model.

Table 4. Drag Coefficients

\begin{tabular}{|c|c|c|c|}
\hline & Pressure & Viscous & $\mathbf{C D}_{\mathbf{D}}$ \\
\hline without mirror & 0,4707 & 0,0330 & $\mathbf{0 , 5 0 3}$ \\
\hline with mirror & 0,5116 & 0,0288 & $\mathbf{0 , 5 3 9}$ \\
\hline with camera & 0,4953 & 0,0265 & $\mathbf{0 , 5 2 1}$ \\
\hline
\end{tabular}

In Figure 6, the pressure distributions on the bus without side mirrors, with side mirrors and cameras are indicted. High-pressure regions appear on the interior of the side mirrors. Low-pressure distribution is observed on camera housing.

\section{Conclusions}

In this study, the drag coefficient of a bus with classic side mirror was found to be $6.6 \%$ higher than the bus without mirror it was determined that using a camera instead of the side mirror decreased the friction coefficient by $3.45 \%$. The side mirrors have been increased the drag coefficient of the bus by 0.036 . The cameras have been increased by 0.018 . As a result, a half gain was achieved in the drag coefficient. The effect of the side mirrors on the bus drag coefficient is a non-negligible amount, and significantly increases fuel consumption. Using camera systems to be placed in smaller volumes instead of side view mirrors reduces the drag coefficient of the bus, resulting in a significant reduction in fuel consumption. Drag coefficient can be further reduced by investigating on the geometric struc- ture of the camera housing or the position of the camera.

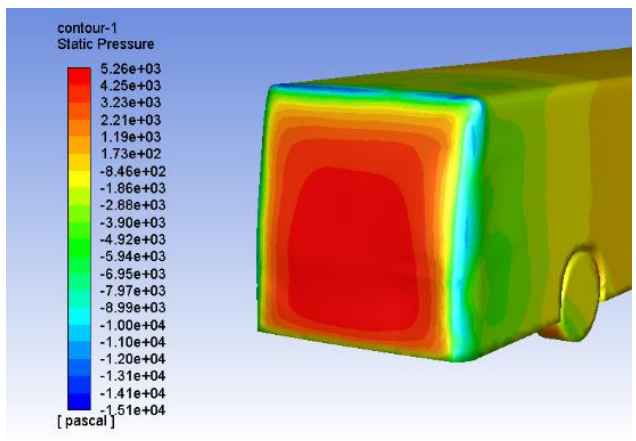

(a)

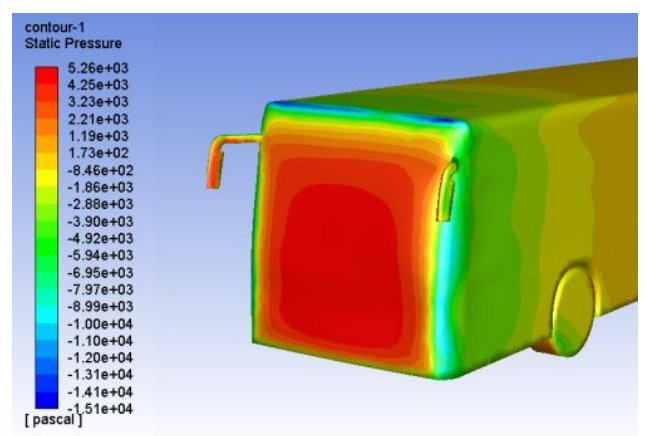

(b)

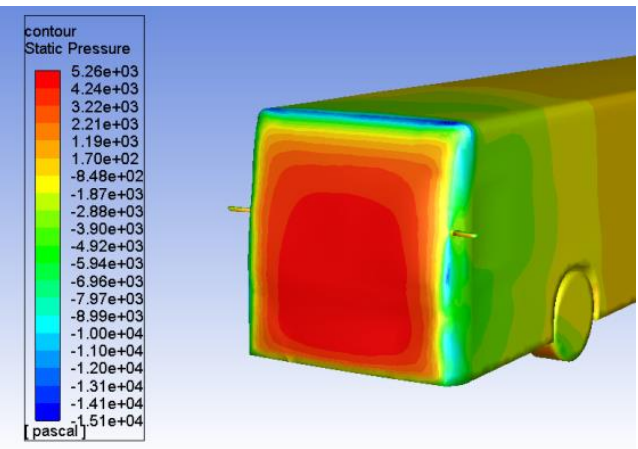

(c)

Fig. 6. Pressure distributions on the bus model (a) without mirror, (b) with mirror, (c) camera

\section{References}

[1] Wang, J., Bartow, W., Moreyra, A., Woyczynski, G., Lefebvre, A., Carrington, E., \& Zha, G. (2014). Low drag automotive mirrors using passive jet flow control. SAE International Journal of Passenger Cars-Mechanical Systems, 7(2014-01-0584), 538-549.

[2] Gan, J., Li, L., Zha, G., \& Czlapinski, C. (2017). Truck rear view mirror drag reduction using passive jet boat tail flow control. SAE Technical Paper (No. 2017-01-1538).

[3] Roy, J. V., Kumar, T. S., Kumar, R. A., Jeeva, S., \& Kumar, V. V. (2018). Aerodynamic design improvement for an inter- 
city bus. International Research Journal of Automotive Technology, 1(4), 85-90.

[4] Ipci D., Yilmaz E., Aysal F.E., Solmaz H. (2015). Numerical investigation on the flow pattern around a land vehicle. Electronic Journal of Machine Technologies 12(2), 51-64.

[5] Gebel M.E., Önaldı S., Korkmaz S., Osmanoğlu S., Özçelik B., Ermurat M., İmal M. (2018). Experimental and numerical investigation of aerodynamic properties of an electrical vehicle. 9th International Automotive Technologies Congress, OTEKON.

[6] J Howell, J., Windsor, S., and Le Good, G. (2006). A Novel test rig for the aerodynamic development of a door mirror. SAE Technical Paper (No. 2006-01-0340).

[7] Hirose, K., Nakagawa, R., Ura, Y., Kawamata, H., Tanaka, H., \& Oshima, M. (2015). Application of prediction formulas to aerodynamic drag reduction of door mirrors. SAE Technical Paper (No. 2015-01-1528).

[8] Buscariolo, F. F., \& Rosilho, V. (2013). Comparative CFD study of outside rearview mirror removal and outside rearview cameras proposals on a current production car. SAE Technical Paper (No. 2013-36-0298).

[9] Katz, J. (2016). Automotive aerodynamics. John Wiley \& Sons.

[10]Lanfrit M. (2005). Best practice guidelines for handling Automotive External Aerodynamics with FLUENT. Darmstadt: Fluent Deutschland GmbH.

[11]ANSYS FLUENT 12.0 Theory Guide - 4.9 Reynolds Stress Model. 